African Crop Science Journal by African Crop Science Society is licensed under a Creative Commons Attribution 3.0 Uganda License. Based on a work at www.ajol.info/ and www.bioline.org.br/cs DOI: http://dx.doi.org/10.4314/acsj.v25i4.2

\title{
SIMULATION DU CLIMAT FUTUR ET DES RENDEMENTS AGRICOLES EN REGION SOUDANO-SAHELIENNE EN REPUBLIQUE DU BENIN
}

\author{
S. KATE, O. TEKA ${ }^{1}$, R.B. CHABI ${ }^{2}$, R. DJIKPO ${ }^{1}$, E. OGOUWALÉ ${ }^{2}$, B.A.H. TENTÉ ${ }^{2}$ et B. SINSIN ${ }^{1}$ \\ Institut National des Recherches Agricoles du Bénin, BP 884, Agonkanmey/Abomey-Calavi, Bénin \\ ${ }^{1}$ Laboratoire d'Ecologie Appliquée, Faculté des Sciences Agronomiques, Université d'Abomey-Calavi, 03 \\ BP1974 Cotonou, Benin \\ ${ }^{2}$ Département de Géographie et Aménagement du Territoire, Faculté des Lettres, Arts et Sciences Humaines, \\ Université d'Abomey Calavi, BP. 526 Cotonou, Bénin \\ Auteur correspondant: oscar_teka@yahoo.fr
}

(Received 1 August, 2017; accepted 29 September, 2017)

\section{RÉSUMÉ}

Le changement climatique est sans équivoque et affecte les systèmes biophysiques. La prédiction des effets futurs du changement climatique constitue un préalable indispensable dans l'élaboration des stratégies d'adaptation et d'atténuation. La présente étude a été initiée pour déterminer les caractéristiques des rendements des principales spéculations agricoles de la région soudano-sahélienne de l'A frique de l'Ouest et plus particulièrement la Commune de Banikoara à l'horizon 2050. La méthodologie adoptée regroupe la collecte des statistiques agricoles et des données météorologiques sur le long terme, le calcul d'indices agro-climatiques et la simulation des rendements au moyen de DSSAT (Decision Support System for Agrotechnology Transfert) et Crop-Model. Des résultats, il apparaît que les températures de la région soudano-sahélienne connaitront une hausse comprise entre $1^{\circ}$ et $3^{\circ} \mathrm{C}$ en référence aux valeurs de la période 1971-2010. La pluviométrie de la saison agricole baissera par rapport à la période de référence selon les scénarios d' augmentation de la température moyenne $\left(\mathrm{de} 1{ }^{\circ} \mathrm{C}, 1,5^{\circ} \mathrm{C}\right.$ et $\left.2^{\circ} \mathrm{C}\right)$. Outre le sorgho pour lequel une amélioration de $9,3 \%$ du rendement a été observée, les rendements des spéculations agricoles baisseront à l'horizon 2050. Le plus fort niveau de baisse de rendement simulé a été enregistré pour le manioc tandis que l'igname a affiché la baisse de rendement le plus faible. En effet, si le manioc affectionne les climats chauds, il exige une certaine humidité qui n'est plus assurée compte tenu du changement climatique. La modification du calendrier agricole et plus précisément l'adaptation des périodes de semis aux perturbations climatiques constatées apparaît comme une nécessité urgente pour garantir la sécurité alimentaire dans la région soudano-sahélienne.

Mots Clés: Indices agro-climatiques, DSSAT, prédiction, Sorghum bicolor

\begin{abstract}
Climate change is unequivocal and affects biophysical systems. Predicting the future effects of climate change is a prerequisite for the development of adaptation and mitigation strategies. This study was carried out to determine the future yields of the main agricultural crops in the Sudano-Sahelian region of West Africa, and especially using Banikoara district of Republic of Benin, for the period up to 2050. The methodology adopted combined the collection of long term agricultural and meteorological data, the calculation of agro-climatic indexes and simulation of crop yields using DSSAT (Decision Support System for Agrotechnology Transfert) and Crop-Model. Results revealed that the temperatures of Sudano-Sahelian region will increase in temperature by about 1 to $3{ }^{\circ} \mathrm{C}$ compared to the reference period 1971-2010. The rainfall of the agricultural season will decline under the
\end{abstract}


assumption of an increase in mean temperature (of $1,1.5$ and $2^{\circ} \mathrm{C}$ ). Apart from sorghum of which the simulated yield will increase about $9,3 \%$, other crops will be characterised by a decline by 2050 of projected yields. The greatest decline in simulated yield was recorded for cassava; while yam showed the lowest decline in yield. Indeed, if cassava has particular affection for hot climates, it requires a certain soil moisture that is no longer guaranteed due to climate change. The shift of agricultural calendar and especially the adaptation of the sowing date to the observed climatic disturbances is urgently needed to ascertain food security insurance in the SudanoSahelian region of Benin.

Key Words: Agro-climatic indexes, DSSAT, prediction, Sorghum bicolor

\section{INTRODUCTION}

L'Afrique subsaharienne est l'une des régions les plus vulnérables au changement climatique (IPCC, 2011). Elle est soumise à d'intenses variabilités des paramètres climatiques qui se manifestent par une mauvaise répartition dans le temps et dans l'espace des précipitations et par une hausse des températures (Lankao, 2010; McCarthy et al., 2010). Le changement climatique aurait comme conséquences la baisse des rendements agricoles et une augmentation des risques d'insécurité alimentaire et nutritionnelle (Newman, 2016.). La recherche de stratégies efficaces d'adaptation et d'atténuation constitue un préalable indispensable pour une assurance de la sécurité alimentaire dans le futur. Alors que l'adaptation suppose l'ajustement des pratiques ou activités pour limiter les effets néfastes et profiter des avantages offerts par le processus du changement climatique, l'atténuation consiste à la création des puits à carbone et la réduction des sources d'émission des gaz à effet de serre (Lal, 2010, IPCC, 2014). La connaissance anticipée des probables effets du changement climatique sur les systèmes de production permettra le développement de stratégies efficaces d'atténuation et d'adaptation (Teka et al., 2013).

$\mathrm{Au}$ Bénin, le secteur agricole, est très affecté par les effets de la variabilité climatique du fait du caractère essentiellement pluvial de l'agriculture (Sarr et al., 2012). Ce secteur constitue, cependant, la base de l'économie béninoise et contribue pour près de $36 \%$ au PIB et $88 \%$ aux recettes d'exportation selon le Ministère de l'Agriculture de l'Elevage et de la pêche (MAEP, 2007) et l'Institut National des Statistiques et d'Analyse Economique (INSAE, 2009). Une attention particulière mérite d'être accordée au secteur agricole pour s'assurer une autosuffisance alimentaire. De plus, les différentes projections futures du climat à travers différents scénarios du $5^{\text {ème }}$ rapport du Groupe d'experts Intergouvernemental sur l'Evolution du Climat (GIEC) ne sont en faveur d'une quelconque amélioration de la productivité agricole. Une forte incertitude et une variabilité des paramètres climatiques principaux tels que les précipitations et la température sont probables (IPCC, 2011). Selon l'Institut National des Statistiques et d'Analyse Economique (INSAE, 2009), 61,1\% de la population du pays vivant en milieu rural dépend de l'agriculture pour sa subsistance. Ainsi, l'intérêt d'élaboration et de la mise en œuvre des mesures d'adaptation pour cette frange de population se justifie à plus d'un titre. Pour l'OMM (Organisation Météorologique Mondiale) et le PNUE (Programme des Nation-Unies pour l'Environnement), la réduction des effets néfastes, directs ou indirects potentiels des changements climatiques sur le système de production agricole passe par la mise en œuvre des mesures adaptatives aussi bien pour les populations que pour les systèmes économiques (OMM et PNUE, 2002).

La région soudano-sahélienne de l'Afrique de l'Ouest et plus particulièrement la Commune de Banikoara au Bénin est une région de forte production de produits vivriers et de rente. Elle est soumise d'ores et déjà au processus du changement climatique (Kate, 2006). Il s'avère nécessaire d'établir des projections 
futures du niveau de vulnérabilité du système de production agricole au changement climatique afin d'anticiper sur les mesures adaptatives à apporter pour pallier les effets néfastes futurs. C'est dans ce contexte que cette Commune a été choisie, pour simuler à l'horizon 2050, les rendements agricoles des principales cultures vivrières et une culture de rente. Les cultures vivrières sont le maïs (Zea mays L.), le sorgho (Sorghum bicolor, (L.), Moench, Meth), le manioc (Manihot esculenta Crantz), le riz (Oryza sativa L.), l'arachide (Arachis hypogaea L.), l'igname (Dioscorea $s p$ ), le soja (Glycine max (L.) Merr.) et le niébé (Vigna unguiculata Walp.). La culture de rente de grande production pratiquée à Banikoara est le coton (Gossypium hirsutum L.).

L'objectif général de cette étude est de déterminer les patrons de rendements des principales spéculations de la région soudanosahélienne en prenant la Commune de Banikoara afin de constituer une base de données utilisables par les décideurs quant à la prise de décisions devant aider les communautés locales à renforcer la résilience de leur système de production face au changement climatique. De façon spécifique, il s'est agi de (i) caractériser le climat futur (température et pluviométrie) de Banikoara à l'horizon 2050, (ii) déterminer la variation des indices agro-climatiques à l'horizon 2050, et (iii) simuler les rendements $\left(\mathrm{kg} \mathrm{ha}^{-1}\right)$ des cultures en 2050 selon le scénario pédologique le plus optimiste.

\section{MATERIEL ET METHODE}

Milieu d'étude. La Commune de Banikoara est située au Nord-Ouest du Département de $1^{\prime}$ Alibori, entre les latitudes $11^{\circ} 02^{\prime}$ et $11^{\circ} 34^{\prime}$ Nord et les longitudes $2^{\circ} 05^{\prime}$ et $2^{\circ} 46^{\prime}$ Est (Fig. 1). Elle couvre une superficie de $4397,2 \mathrm{~km}^{2}$ dont environ $49 \%$ de terres cultivables et 50 $\%$ d'aires protégées (Parc National du W du Niger et la zone cynégétique de l'Atacora). Elle compte environ 246575 habitants. La densité de population est estimée à $34,7 \mathrm{hab} \mathrm{km}^{-2}$
(RGPH, 2013). Les principaux groupes ethniques sont Baatombu, Fulbé ou Peulh, Dendi, Yoruba et Fon. Quant à la religion, les plus pratiquées sont l'islam $(51,7 \%)$, la religion traditionnelle $(34,4 \%)$, le christianisme dont le catholicisme $(7,0 \%)$ et le protestantisme $(1,1 \%)$, les autres religions $(5,8 \%)$. L'agriculture et l'élevage sont les principales activités économiques exercées par les populations de Banikoara.

Collectes des données. Les données climatiques (précipitation, humidité relative, températures) sur la période allant de 1971 à 2010, ont été collectées à l'Agence pour la Sécurité de la Navigation Aérienne (ASECNA) de Cotonou pour la Commune de Banikoara. Les statistiques annuelles des productions agricoles des spéculations retenues entre 1971 et 2010, ont été obtenues au Ministère de l'Agriculture, de l'Elevage et de la Pêche (MAEP), à l'Institut National de Statistique et de l'Analyse Economique (INSAE) et à l'Office Nationale d'Appui à la Sécurité Alimentaire (ONASA). Ces statistiques sont relatives aux productions (en tonnes), superficies emblavées (en ha) et rendements (en $\left.\mathrm{kg} \mathrm{ha}^{-1}\right)$.

\section{Analyse des données}

Scénarios utilisés et suppositions de base. Dans la présente étude, douze (12) scénarios de base (Tableau 1) composites issus des variables de températures (augmentation de la température moyenne de $1^{\circ} \mathrm{C}, 1,5^{\circ} \mathrm{C}$ et $2^{\circ} \mathrm{C}$ ) et de la durée de la saison de croissance (en lieu et place de la pluie) ont été formulés et retenus en fonction des considérations : (i) des amplitudes de changement simulées par les modèles climatiques; (ii) des tendances générales des variables climatiques observées par les stations météorologiques du milieu d'étude et (iii) du choix raisonnable d'approcher l'analyse du risque alimentaire par les scenarii pessimistes (principe de précaution). 


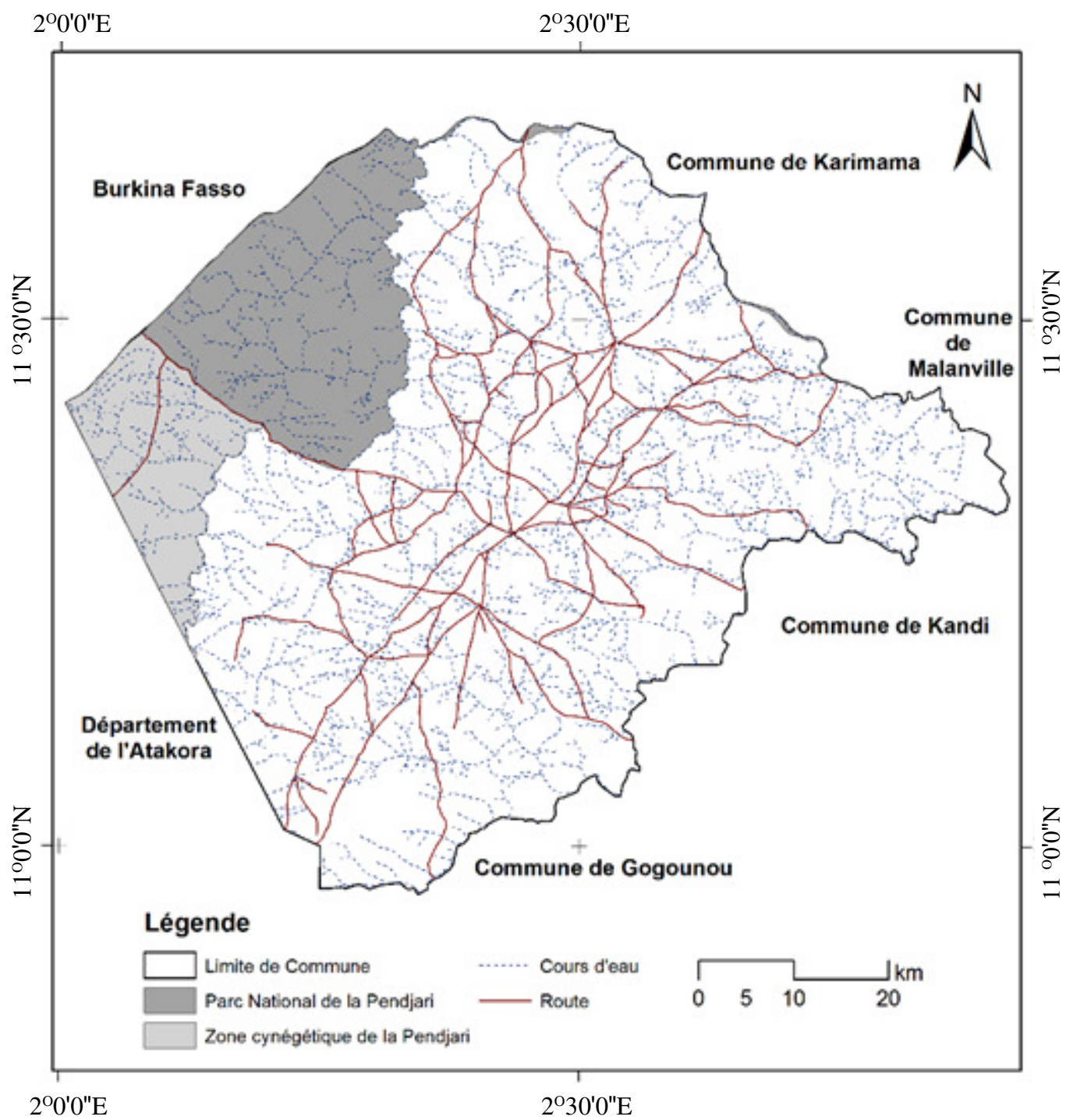

Figure 1. Situation géographique et administrative de la Commune de Banikoara.

TABLEAU 1. Scénarios climatiques dans la zone agro-pastorale de production cotonnière à l'horizon 2050

\begin{tabular}{llll}
\hline Scénarios & \multicolumn{3}{c}{ Scénarios thermiques } \\
\cline { 2 - 4 } Durée de la Saison de croissance & $+1{ }^{\circ} \mathrm{C}$ & $+1,5{ }^{\circ} \mathrm{C}$ & $+2{ }^{\circ} \mathrm{C}$ \\
\hline$-10 \%$ & Scénario certain & Scénario certain & Scénario critique \\
$-15 \%$ & Scénario certain & Scénario probable & Scénario critique \\
$-20 \%$ & Scénario probable & Scénario critique & Scénario extrême \\
$-25 \%$ & Scénario critique & Scénario extrême & Scénario extrême \\
\hline
\end{tabular}


Par la suite, trois types de sols : (i) sol ferrugineux à sesquioxydes et à concrétions sur granito-gneiss avec $\mathrm{pH}$ neutre à basique (7-8), désigné par S1 dans la présente étude; (ii) sol formé d'une superposition de S1, de sol ferralitique à concrétions et cuirasses de texture argilo-sableux et horizons évolués, caractérisé par un drainage interne et un $\mathrm{pH}$ neutre à basique (7-8), désigné par $\mathrm{S} 2$ dans la présente étude; (iii) sol hydromorphe peu humifère sur alluvions, à pseudogley à tâches, désigné par S3 ont été déterminés (Van Diepen et al., 1988).

La tendance de l'état de référence du facteur pédologique dans la simulation du rendement futur est construite comme dans le Tableau 2.

Après la combinaison des scénarios climatiques de base et des suppositions pédologiques, trente-six (36) scénarios de rendements possibles par culture ont été générés.

Détermination de la saison de croissance. La détermination des caractéristiques de la saison de croissance (durée, début et fin) dans le Crop-Model (Jones et al., 2003) s'est faite à travers le calcul de l'Evapotranspiration Potentielle (ETP) mensuelle selon Sumner et Jacobs (2005). Les valeurs mensuelles de l'ETP ont été extrapolées en valeurs journalières (Tableau 3) utilisable par le Crop-Model. Il en est de même des hauteurs des précipitations.

Détermination de la biomasse et du rendement par culture. La production de biomasse nette est tirée de FAO (1978). Elle tient compte du taux maximum de production de biomasse brute pendant la saison de croissance en $\mathrm{kg} \mathrm{ha}^{-1} \mathrm{~J}^{-1}$; du coefficient de transpiration de la plante (McCree, 1974) et de la longueur de la saison de croissance en jour. En raison de la difficulté à quantifier la plupart des contraintes agro-climatiques qui réduisent les rendements des cultures, le logiciel Decision Support System for Agrotechnology Transfert (Hoogenboom et al., 2015) DSSAT
V4.0 a été utilisé pour n'estimer que trois d'entre elles à savoir le stress hydrique; la contrainte de travail et la qualité du sol.

La projection des paramètres climatiques à l'horizon 2050 s'est faite en tenant compte de scénarios empiriques (analogues sec et humide) et des données extraites du HadCM2 et du CSIRO-Tr sur la base des scénarios SRESA2. Ces données ont été introduites dans le DSSAT V4.0 pour l'estimation des rendements potentiels des cultures étudiées. Les variables d'entrée du Crop-Model concernent les données climatiques (les informations sur les spéculations choisies et les conditions du sol. Les critères d'appréciation des rendements agricoles sont ceux conçus par Komolafe et al. (1980) qui ont donné les minima et maxima des rendements de différentes cultures.

\section{RESULTATS}

Climat futur et évolution des indices agroclimatiques à Banikoara. Les statuts des températures mensuelles de Banikoara à l'horizon 2050 (Fig. 2) ont montré une augmentation des températures minimales $\left(0,5^{\circ} \mathrm{C}-2^{\circ} \mathrm{C}\right)$ et maximales $\left(1,5^{\circ} \mathrm{C}-3^{\circ} \mathrm{C}\right)$ selon les scénarios.

Les indices des températures minimales et maximales à l'horizon 2050 (Fig. 3) ont montré que la magnitude des anomalies de température minimale moyenne sera de $-2,08^{\circ}$ à $+2,5^{\circ} \mathrm{C}$ et celle des anomalies de température maximale moyenne sera de $-1,76^{\circ}$ à $2,12^{\circ} \mathrm{C}$.

Les statuts de précipitations (Fig. 4) révèlent une grande fluctuation. Les pluies des mois de la saison culturale (avril-octobre) connaîtront des modifications. Les hauteurs des précipitations de la saison culturale diminueront $17 \%$ par rapport à la période de référence (1971-2010). Un tel contexte affectera les indices agro-climatiques dans la Commune de Banikoara.

Excepté le nombre de jours intermédiaires (NJI) qui a connu une augmentation de quatre (04) jours, les autres indices agro-climatiques 
TABLEAU 2. Suppositions de l'état de la qualité des sols pour la simulation des rendements des cultures

\begin{tabular}{|c|c|c|c|c|}
\hline \multirow[t]{2}{*}{ Potentialités agronomiques } & \multirow[t]{2}{*}{ Actuelles } & S1 & $\mathrm{S} 2$ & S3 \\
\hline & & $\begin{array}{l}\text { Moyenne à bonne bonne } \\
\text { pour toute culture }\end{array}$ & $\begin{array}{l}\text { Excellent milieu de } \\
\text { croissance, }\end{array}$ & Très fertilebonne pour toute culture \\
\hline & $\begin{array}{l}\text { Situation future sans amélioration } \\
\text { des conditions du sol }\end{array}$ & Infertile & Moyenne & Moyenne à bonne \\
\hline & $\begin{array}{l}\text { Situation future avec amélioration } \\
\text { des conditions du sol }\end{array}$ & Bonne & Bonne à très bonne & Très bonne \\
\hline \multirow[t]{3}{*}{ Indice cultural } & Actuel & 0,75 & 1,00 & 1,00 \\
\hline & $\begin{array}{l}\text { Situation future sans amélioration } \\
\text { des conditions du sol }\end{array}$ & Moins de 0.5 & 0,75 & $0,75-1,00$ \\
\hline & $\begin{array}{l}\text { Situation future avec amélioration } \\
\text { des conditions du sol }\end{array}$ & $0,75-1,00$ & 1,00 & 1,00 \\
\hline
\end{tabular}

Un sol amélioré équivaut à un sol ayant bénéficié des apports en engrais organiques ou chimiques 
TABLEAU 3. Variation des indices agro-climatiques (1971-2010 et 2050) à Banikoara

\begin{tabular}{llcc}
\hline & Climat 1971-2010 & Climat futur (2050) & Ecart en jours \\
\hline Nombre de jours secs & 206 & 199 & -7 \\
Nombre de jours intermédiaires & 63 & 67 & +4 \\
Nombre de jours humides & 126 & 116 & -10 \\
Début de la saison de croissance & $17 \mathrm{Mai}$ & $11 \mathrm{Mai}$ & -6 \\
Fin de la saison de croissance & 19 Oct & 10 Oct & -9 \\
Durée de la saison de croissance & 190 & 173 & -17 \\
\hline
\end{tabular}
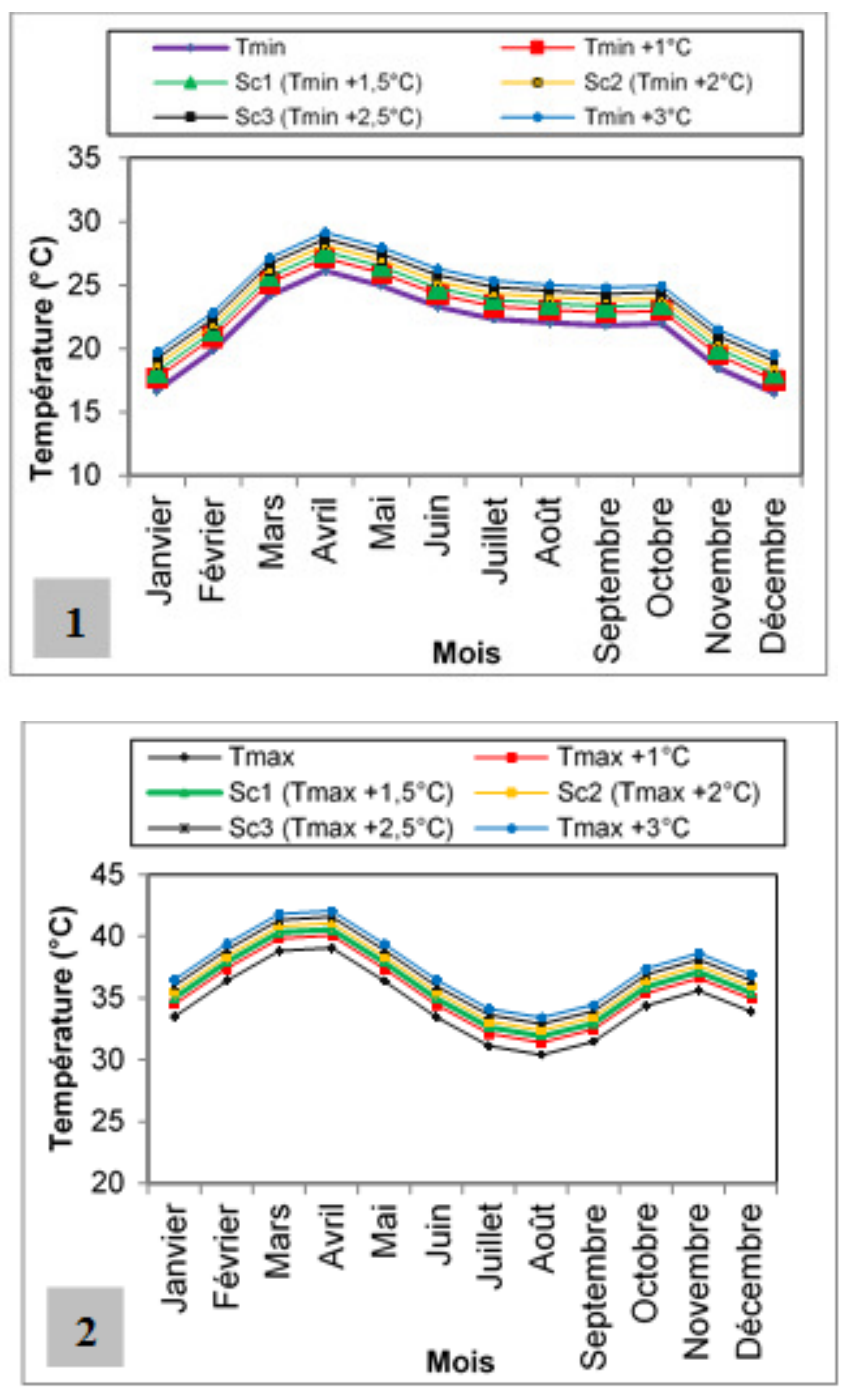

Tmin : Température minimale de la période de référence 1981-2012; Tmax : Température maximale de la période de référence 1981-2012; Sc : scénario

Figure 2. Evolution future des températures minimales (1) et maximales (2) mensuelles dans la Commune de Banikoara à l'horizon 2050 par rapport à celles de la période .1981-2012. 

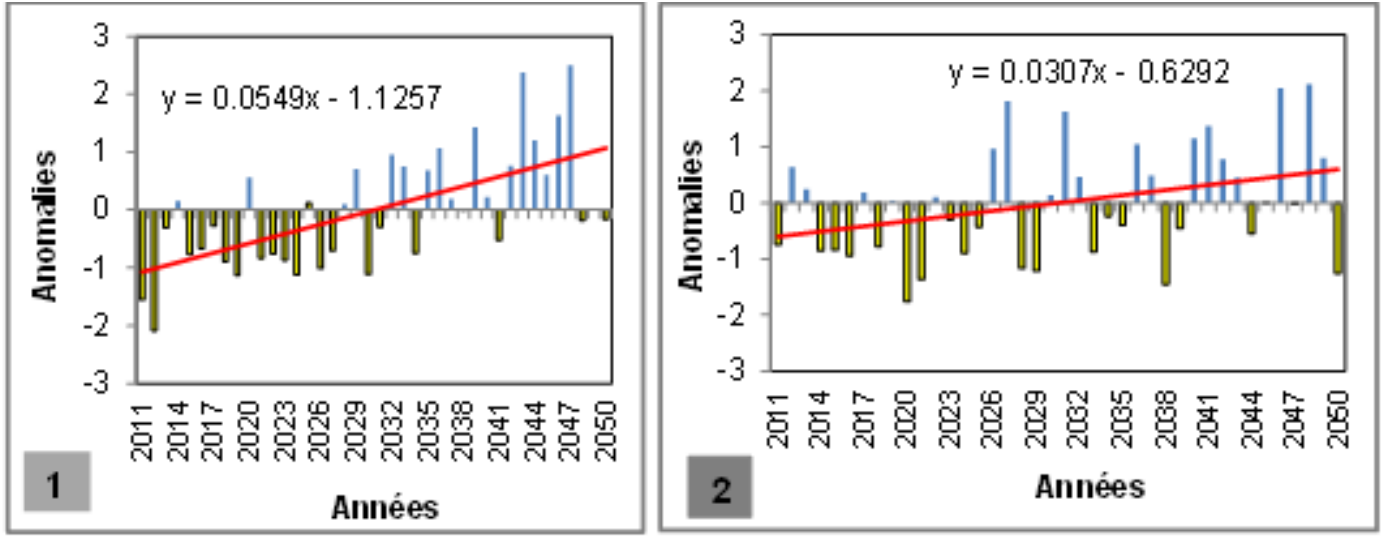

Figure 3. Indices des températures minimales (1) et maximales (2) à l'horizon 2050.

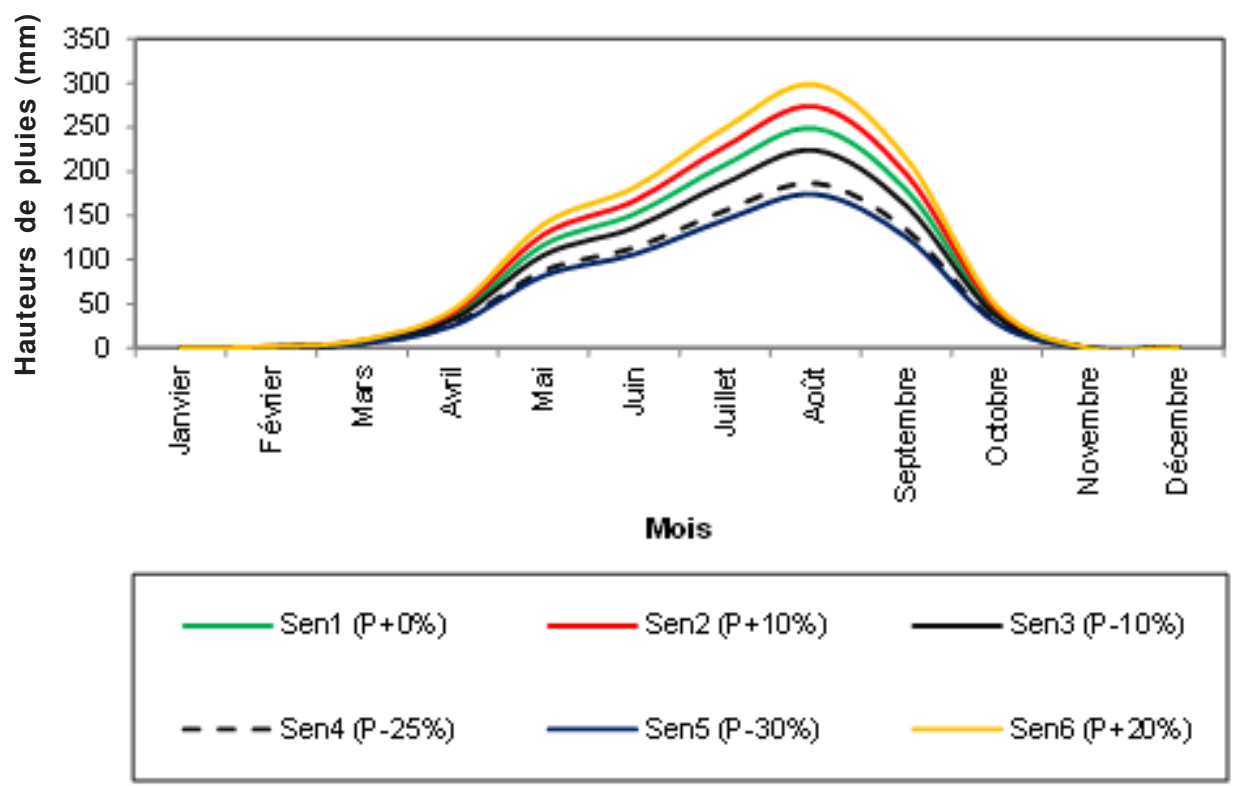

Figure 4. Scénarios pluviométriques saisonniers dans la Commune de Banikoara.

généraux de la Commune de Banikoara (Tableau 3) se sont dépréciés à l'horizon 2050 en référence aux valeurs obtenues pour la période 1971-2010. Ainsi, la durée de la saison de croissance (DuSC) a baissé de 17 jours, le début de la saison de croissance (DSC) a diminué de 06 jours, la fin de la saison de croissance (FSC) s'est raccourcie de 09 jours, et le nombre de jours humides $(\mathrm{NJH})$ a diminué de 10 jours. Ces réductions sont susceptibles d'impacter le développement des cultures et par conséquent leurs rendements agricoles.

\section{Rendements moyens simulés à l'horizon} 2050 selon les scénarios pédologiques les plus optimistes. Les rendements des cultures ont été très fluctuants suite aux conditions agro-climatiques futures (Tableau 4) conséquemment à la variabilité des indices agro-climatiques (Fig. 5). 
TABLEAU 4. Rendements $\left(\mathrm{kg} \mathrm{ha}^{-1}\right)$ des cultures en 2050 dans la Commune de Banikoara selon le scénario pédoclimatique le plus optimiste ("'-10\% DuSC, $+1,5^{\circ} \mathrm{C}, \mathrm{S} 3$ ")

\begin{tabular}{|c|c|c|c|c|c|c|}
\hline \multirow[t]{2}{*}{ Cultures } & \multicolumn{2}{|c|}{ Rendement actuel } & \multicolumn{2}{|c|}{ Rendement futur } & \multirow[t]{2}{*}{ Ecart } & \multirow[t]{2}{*}{ De variation $(\%)$} \\
\hline & Valeur & Niveau & Valeur & Niveau & & \\
\hline Arachide & 1159,4 & Très élevé & 1042,15 & Fort & $-17,25$ & $-1,5$ \\
\hline Coton & 1192 & Elevé & 1029,02 & Fort & $-162,98$ & $-13,7$ \\
\hline Niébé & 923,6 & Très élevé & 864,96 & Très fort & $-58,64$ & $-6,3$ \\
\hline Manioc & 6409 & Faible & 4696,84 & Très faible & $-1712,16$ & $-26,7$ \\
\hline Igname & 8178,5 & Moyen & 8162,81 & Moyen & $-15,69$ & $-0,2$ \\
\hline Riz & 2891,6 & Très élevé & 2557,85 & Très fort & $-333,75$ & $-11,5$ \\
\hline Maïs & 1185 & Elevé & 1000,05 & Moyen & $-184,95$ & $-15,6$ \\
\hline Sorgho & 959,6 & Très élevé & 1048,89 & Très fort & 89,29 & $+9,3$ \\
\hline Soja & 634,2 & Elevé & 563,5 & Moyen & $-70,7$ & $-11,1$ \\
\hline
\end{tabular}
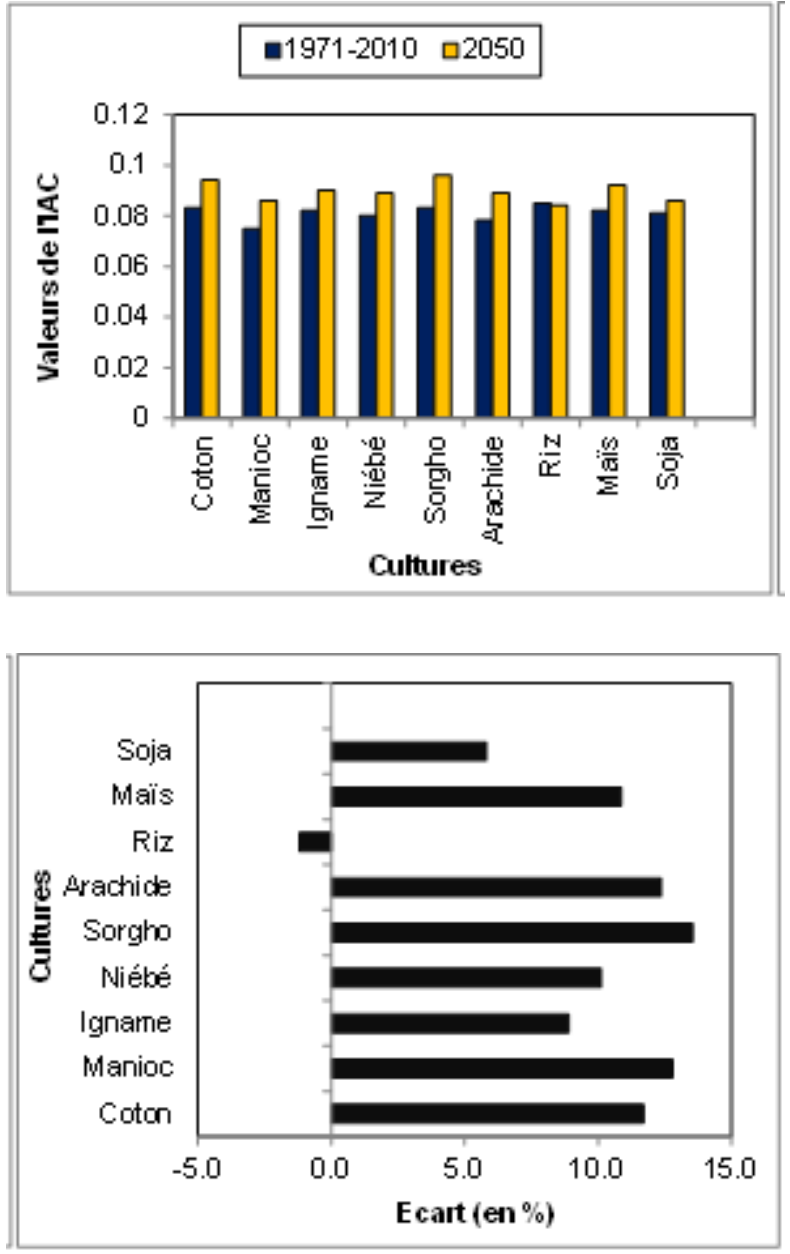

Figure 5. Indices agro-climatiques pour la période 1971-2010 et projection en 2050 et écart entre 1971-2010 et 2050 en pourcentage. 
Outre le sorgho qui connaîtra une amélioration de 9,3\% de son rendement, les rendements des autres cultures vont systématiquement baisser de $(-26,7 \%$ pour le manioc à $-0,2 \%$ pour l'igname). Toutefois, les rendements resteront très forts pour le niébé (923,6-864,96 $\left.\mathrm{kg} \mathrm{ha}^{-1}\right)$, le sorgho $(959,6-$ $\left.1048,89 \mathrm{~kg} \mathrm{ha}^{-1}\right)$ et le riz $(2891,6-2557,85 \mathrm{~kg}$ $\left.\mathrm{ha}^{-1}\right)$. Les rendements seront forts pour l'arachide (1159,4-1042,15 $\left.\mathrm{kg} \mathrm{ha}^{-1}\right)$ et le coton (1192-1029,02 $\left.\mathrm{kg} \mathrm{ha}^{-1}\right)$ tandis qu'ils seront moyens pour le maïs (1185-1000,05 $\mathrm{kg} \mathrm{ha}^{-1}$ ), l'igname $\left(8178,5-8162,81 \mathrm{~kg} \mathrm{ha}^{-1}\right)$ et le soja $\left(634,2-563,5 \mathrm{~kg} \mathrm{ha}^{-1}\right)$.

\section{DISCUSSION}

Climats futurs de Banikoara. Les températures ont connu une augmentation et confirme le processus de réchauffement climatique dans la région ouest-africaine (Newman, 2016). En plus de cette tendance à la hausse de température, l'alternance des anomalies thermométriques positives et négatives vient illustrer la fluctuation thermométrique caractéristique du milieu d'étude (Roudier et al., 2011). En d'autres termes, les températures sont de plus en plus élevées. Cette hausse de température devrait rester dans une certaine limite en fonction des types de plantes (cultures) pour permettre une activation des réactions physico-chimiques du métabolisme (Garau et al., 2007). Trop élevée, la hausse de température engendrerait une détérioration des structures vivantes dans lesquelles s'effectuent lesdites réactions chimiques (Abou-Arab et al., 2010).

Les régimes pluviométriques futurs ne connaîtront pas de modification profonde puisque le régime uni-modal sera maintenu. Cependant, les pluies des mois de la saison agricole (Avril-Octobre) connaîtront des modifications. La pluviométrie de cette période va décroitre par rapport à la période de référence (1971-2010). Autrement dit, les hauteurs de pluie pourraient baisser phases sensibles de développement des cultures (début, milieu et fin). Mieux une augmentation des hauteurs de pluie en Août-Septembre sera enregistrée en fin de saison de croissance. En d'autres termes, à l'horizon 2050, la Commune de Banikoara sera marquée par des modifications mensuelles et saisonnières dans le contexte des changements climatiques prévus. La diminution du stock d'eau dans le sol pendant la phase critique détermine le rendement des cultures (Lobell et al., 2011). Aussi une augmentation thermique pourrait être source de stress supplémentaire pour les plantes et les sols, impactant de fait les rendements agricoles dans les différentes zones agro-écologiques (Ferris et al., 1998 ; Issa, 2012).

Les variations des paramètres climatiques sont alors susceptibles d'agir sur les rendements et la qualité des récoltes. Celles-ci peuvent être traduites par la modification des indices agro-climatiques.

\section{Variation des indices agro-climatiques et} rendements des cultures. Les indices agroclimatiques déterminent non seulement les patrons de rendement mais aussi la qualité des cultures. Ainsi une augmentation des indices agro-climatiques (IAC) conséquemment à une élévation de la température entraverait le métabolisme et la croissance de bon nombre de cultures céréalières (FAO, 1997 ; Ferris $e t$ al., 1998). Les IACs calculés pour la Commune de Banikoara connaîtront tous une modification pour la quasi-totalité des cultures témoignant un stress thermique supplémentaire avec un risque élevé de réduction des rendements simulés des cultures. Les températures minimales plus importantes ainsi que les températures maximales plus élevées nuiront généralement à de nombreuses cultures (Kruk et al., 2006). Les températures étant déjà proches des valeurs maximales tolérées pour la plupart des cultures testées, il est évident que les rendements des cultures diminueront, même avec une hausse minime des températures associée à l'altération de l'état hydrique qui va engendrer une modification 
de la matière organique des sols déjà irrationnellement exploités.

D'un autre côté, la hausse de température pourrait aussi être source de prolifération et propagation des adventices et des ravageurs des cultures (Parry, 1990 ; FAO, 2002). Ceci pourrait affaiblir davantage le potentiel de productivité des cultures. La hausse de température associée au raccourcissement de la période active de végétation favorise, en régions tropicales, le développement rapide des mauvaises herbes, des maladies cryptogamiques et des ravageurs de culture (FAO, 2002; Singh et al., 2012). En conséquence, les parties consommables des cultures (grains, feuilles, tiges et racines) seront sujets à des attaques vigoureuses. En conséquence, la possibilité de croissance normale, la photosynthèse et la fonction respiratoire des cultures se trouvent perturbées. Le risque de pertes de récolte devient élevé.

Dans la Commune de Banikoara, le scénario "réduction de $10 \%$ de la DuSC avec une augmentation de température de $1,5^{\circ} \mathrm{C}$ sur sol de type S3" est plausible car, non seulement ses constituants sont très probables lorsqu'on se réfère aux projections de tous les modèles climatiques sur les régions intertropicales, mais aussi les amplitudes de changement considérées ont déjà été observées dans les séries chronologiques depuis les années 1970. L'ampleur de la diminution des rendements varie suivant les plantes cultivées (Paeth et al., 2008). Dans le cas des cultures investiguées, le manioc $(-26,7 \%)$, le maïs $(-15,6 \%)$ et le coton $(-13,7 \%)$ seront les plus affectés. Au Bénin, le manioc et le maïs constituent la base de l'alimentation humaine. Ainsi, si le contexte actuel de mise en valeur des terres se maintient, les productions de ces principales cultures alimentaires vont baisser considérablement. Celles de l'igname, du sorgho et du niébé qui représentent les aliments de base des populations de la Commune de Banikoara vont tout de même chuter (ONASA, 2008). Cette situation présage, de toute évidence, une précarité alimentaire dans le contexte des changements climatiques et appelle au développement et à l'application des mesures d'adaptation.

Le rendement du coton va décrô̂tre significativement induisant soit un changement dans la politique de diversification des produits d'exportation soit un surdosage en intrants chimiques qui pourrait par ricochet engendrer des conséquences graves à l'environnement comme la dégradation des sols et la pollution des eaux. Les ressources agropastorales de la Commune de Banikoara seront certainement fragilisées.

\section{CONCLUSION}

La présente étude a mis en exergue la variabilité climatique et ses probables conséquences sur les rendements agricoles à l'horizon 2050 dans la Commune de Banikoara au Bénin. D’une manière générale, les rendements des différentes spéculations ont été très fluctuants entre 1996-1997 et 2011-2012 et connaîtront une baisse à l'horizon 2050 compte de la dépréciation des indices agro-climatiques. Le manioc est la culture dont le rendement sera le plus impacté négativement par le processus de variabilité climatique dans le milieu d'étude. Par ailleurs, les conditions agro-climatiques futures ont été identifiées pouvant être très favorables pour le sorgho avec une amélioration du rendement de l'ordre de $+9,3 \%$ (diminution de $10 \%$ avec une augmentation thermique de $1,5^{\circ} \mathrm{C}$ ). Face à cette situation, il apparaît nécessaire d'adapter le calendrier agricole et plus précisément les périodes de semis des différentes spéculations aux perturbations climatiques constatées dans la Commune de Banikoara pour garantir dans le futur la sécurité alimentaire dans la région soudano-sahélienne.

\section{REMERCIEMENT}

Les auteurs remercient l'Institut National des Recherches Agricoles du Bénin (INRAB) et le Le Centre International pour le Développement des Fertilisants (IFDC) pour avoir 
respectivement supporté et financé la présente étude. Ils tiennent à remercier les anonymes évaluateurs pour leurs précieuses contributions qui ont permis d'améliorer de manière significative la qualité scientifique du manuscrit.

\section{RÉFÉRENCES BIBLIOGRAPHIQUES}

Abou-Arab, A.E., Abou-Arab, A.A. and AbuSalem, M.F. 2010. Physico-chemical assessment of natural sweeteners steviosides produced from Stevia rebaudiana Bertoni plant. African Journal of Food Science 4(5):269-281.

FAO. 1978. Forestry for Local Community Development Food and Agriculture Organization of the United Nation (FAO), FAO Forestry paper, No 7, Rome, Italy.

FAO. 1997. FAO database on introduced aquatic species, FAO, Rome, Italy.

FAO. 2002. Food insecurity: When people must live with hunger and fear starvation. The state of food insecurity in the world 2002. FAO. Rome, Italy. 214pp.

Ferris, R., Ellis, R.H., Wheeler, T.R. and Hadley, P. 1998. Effect of high temperature stress at anthesis on grain yield and biomass of field-grown crops of wheat. Annals of Botany 82(5):631-639.

Garau, M.C., Simal, S., Rossello, C. and Femenia, A. 2007. Effect of air-drying temperature on physico-chemical properties of dietary fibre and antioxidant capacity of orange (Citrus aurantium $v$. Canoneta) by-products. Food Chemistry 104(3):1014-1024.

Hoogenboom, G., Jones, P. W., Wilkens, P.W., Porter, C.H., Boote, K.J., Hunt, L.A., Singh, U., Lizaso, J. I., White, J.W., Uryasev, O., Ogoshi, R., Koo, J., Shelia, V. and Tsuji, G.Y. 2015. Decision Support System for Agrotechnology Transfert (DSSAT) Version 4.0 (http://dssat.net). DSSAT Fundation, Professor, Washington.

INSAE. 2009. Tableau de bord Economique et Social. Institut National des Statistiques et d'Analyse Economique. $4^{\text {ème }}$ trimestre, N8 (Février 2009), Cotonou, Bénin.

IPCC. 2011. Mitigation, Climate change. IPCC special report on renewable energy sources and climate change mitigation.

Issa, M.S. 1995. Impacts potentiels d'un changement climatique dû au doublement du CO2 atmosphérique sur l'agriculture en République du Bénin. DESS Université Senghor d'Alexandrie. 113pp.

Jones, P.W., Hoogenboom, G., Porter, C.H., Boote, K.J., Batchelor, W.D., Hunt, L.A., Wilkens, P.W., Singh, U., Gijsman, A.J. and Ritchie, J.T. 2003. DSSAT cropping System Model. European Journal of Agronomy 18:235-265.

Komolafe, O.O., Jarrett, O. and Neil J.C. 1980. Feline calicivirus induced polypeptides. Microbios 27 (109110):185192.

Kate S. 2016. Effets des changements climatiques sur l'environnement agricole et mesures d'adaptation en zone agropastorale de production cotonnière dans la Commune de Banikoara en République du Bénin. Faculté des Sciences Agronomiques, Université d'Abomey-Calavi, Bénin. Thèse de doctorat, 156pp.

Kruk, B., Insausti, P., Razul, A. and Benech Arnold, R. 2006. Light and thermal environments as modified by a wheat crop: effects on weed seed germination. Journal of Applied Ecology 43(2):227-236.

Lal, R. 2010. Beyond Copenhagen: mitigating climate change and achieving food security through soil carbon sequestration. Food Security 2(2):169-177.

Lankoa, P.R. 2010. Water in Mexico city: what will climate change bring to its history of water-related hazards and vulnerabilities? Environ Urban 22:157-178.

Lobell, D.B., Schlenker, W. and CostaRoberts, J. 2011. Climate trends and global crop production since 1980. Science 333(6042):616-620.

MAEP. 2007. Ministère de l'Agriculture de l'Elevage et de la Pêche. Rapport national 
sur l'état des ressources phytogénétiques pour l'alimentation et l'agriculture. Deuxième rapport, Cotonou, Bénin. 57pp. McCarthy, M.P., Best, M.J. and Betts, R.A. 2010. Climate change in cities due to global warming and urban effects. Geophysical Research Letters 37:L09705. doi: 10.1029/ 2010GL042845

McCree, K.J. 1974. Equations for the rate of dark respiration of white clover and grain sorghum, as functions of dry weight, photosynthetic rate, and temperature. Crop Science, DOI:10.2135/cropsci1974.0011 183X001400040005X.

Newman, T.P. 2016. Tracking the release of IPCC AR5 on Twitter: Users, comments, and sources following the release of the Working Group I Summary for Policy makers. Public Understanding of Science, 0963662516628477.

OMM et PNUE. 2002. Organisation Mondiale de la Météorologie (OMM) et Programme des Nations Unies pour l'Environnement (PNUE). Bilan des changements climatiques 2001. Rapport de synthèse. 204pp.

ONASA. 2008. Evaluation de la production vivrière 2008 et des perspectives. Rapport de l'Office National pour la Sécurité Alimentaire (ONASA), Cotonou, Bénin. 52pp.

Paeth, H., Capo-Chichi, A. and Endlicher, W. 2008. Climate change and food security in tropical West Africa: A dynamic-statistical modelling approach. Erdkunde. pp. 101115.

Parry, M. 1990. Climate Change and world Agriculture. Earth scan publication. Ltd, London. England. 151pp.
RGPH4. 2013. Que retenir des effectifs de population en 2013? Institut National de la Statistique et de l'Analyse Economique (INSAE). Direction des Etudes Démographiques/Ministère du Développement, de l'Analyse Economique et de la Prospective. Bénin. 37pp.

Roudier, P., Sultan, B., Quirion, P. and Berg, A. 2011. The impact of future climate change on West African crop yields: What does the recent literature say? Global Environmental Change 21(3):1073-1083.

Sarr, B., Atta, S. and Kafando, L. 2012. Revue des indices climatiques utilisés dans les systèmes d'assurances agricoles indicielles en Afrique. Sécheresse 23 : 255-60.

Singh, P., Boote, K.J. and Kumar, U. 2012. Evaluation of genetic traits for improving: Productivity and adaptation of groundnut to climate change in India. Journal of Agronomy and Crop Science 198: 399-413.

Sumner, D.M. and Jacobs, J.M. 2005. Utility of Penman-Monteith, Priestley-Taylor, reference evapotranspiration, and pan evaporation methods to estimate pasture evapotranspiration. Journal of Hydrology 308(1):81-104.

Teka, O., Houessou, GL., Oumorou, M., Vogt, J. and Sinsin, B. 2013. An assessment of climate variation risks on agricultural production: perceptions and adaptation options in Benin. International Journal of Climate Change Strategies and Management 5(2):166-180.

Van Diepen, C.A., Rappoldt, C., Wolf, J. and Van Keulen, H. 1988. Crop growth simulation model WOFOST. Documentation Version 4.1, SOW-88-01, Wageningen, The Netherlands Centre for World Food Studies. 299pp. 